\title{
A general solution of the Fekete-Szegö problem
}

Jacek Dziok ${ }^{*}$

${ }^{*}$ Correspondence:

jdziok@univ.rzeszow.pl

Institute of Mathematics, University

of Rzeszów, Rzeszów, 35-310, Poland

\begin{abstract}
In the paper we introduce general classes of analytic functions defined by the Hadamard product. The Fekete-Szegö problem is completely solved in these classes of functions. Some consequences of the main results for new or well-known classes of functions are also pointed out.
\end{abstract}

MSC: 30C45; 30C50; 30C55

Keywords: analytic functions; Fekete-Szegö problem; subordination; Hadamard product

\section{Introduction}

Let $\widetilde{\mathcal{A}}$ denote the class of functions which are analytic in $\mathcal{U}=\{z \in \mathbb{C}:|z|<1\}$ and let $\mathcal{A}$ denote the class of functions $f \in \widetilde{\mathcal{A}}$ normalized by $f(0)=f^{\prime}(0)-1=0$. Each function $f \in \mathcal{A}$ can be expressed as

$$
f(z)=z+\sum_{n=2}^{\infty} a_{n} z^{n} \quad(z \in \mathcal{U})
$$

By $\mathcal{S}$, we denote the class of functions $f \in \mathcal{A}$, which are univalent in $\mathcal{U}$.

A typical problem in geometric function theory is to study a functional made up of combinations of the coefficients of the original function. Usually, there is a parameter over which the extremal value of the functional is needed. The paper deals with one important functional of this type: the Fekete-Szegö functional. The classical Fekete-Szegö functional is defined by

$$
\Lambda_{\mu}(f)=a_{3}-\mu a_{2}^{2} \quad(0<\mu<1)
$$

and it is derived from the Fekete-Szegö inequality. The problem of maximizing the absolute value of the functional $\Lambda_{\mu}$ in subclasses of normalized functions is called the FeketeSzegö problem. The mathematicians who introduced the functional, M. Fekete and G. Szegö [1], were able to bound the classical functional in the class $\mathcal{S}$ by $1+2 \exp \left\{\frac{-2 \mu}{1-\mu}\right\}$. Later Pfluger [2] used Jenkin's method to show that this result holds for complex $\mu$ such that $\operatorname{Re} \frac{\mu}{1-\mu} \geq 0$. Keogh and Merkes [3] obtained the solution of the Fekete-Szegö problem for the class of close-to-convex functions. Ma and Minda [4,5] gave a complete answer to the Fekete-Szegö problem for the classes of strongly close-to-convex functions and strongly 
starlike functions. In the literature, there exists a large number of results about inequalities for $\Lambda_{\mu}(f)$ corresponding to various subclasses of $\mathcal{A}$ (see, for instance, [1-23]).

In the paper, we consider the classes of functions which generalize these subclasses of functions.

We say that a function $g \in \widetilde{\mathcal{A}}$ is subordinate to a function $G \in \widetilde{\mathcal{A}}$, and write $g(z) \prec G(z)$ (or simply $g \prec G$ ), if and only if there exists a function

$$
\omega \in \Omega:=\{\omega \in \tilde{\mathcal{A}}:|\omega(z)| \leq|z|(z \in \mathcal{U})\},
$$

such that $g=G \circ \omega$. In particular, if $G$ is univalent in $\mathcal{U}$ we have the following equivalence:

$$
g(z) \prec G(z) \quad \Longleftrightarrow \quad[g(0)=G(0) \wedge g(\mathcal{U}) \subset G(\mathcal{U})] .
$$

For functions $f, g \in \mathcal{A}$ of the forms

$$
f(z)=\sum_{n=1}^{\infty} a_{n} z^{n} \quad \text { and } \quad g(z)=\sum_{n=1}^{\infty} b_{n} z^{n},
$$

by $f * g$ we denote the Hadamard product (or convolution) of $f$ and $g$, defined by

$$
(f * g)(z)=\sum_{n=1}^{\infty} a_{n} b_{n} z^{n} \quad(z \in \mathcal{U})
$$

Let $\alpha$ be complex parameter and let $\Phi=(\phi, \varphi), \Psi=(\psi, \chi), \mathrm{P}=(p, q) \in \widetilde{\mathcal{A}} \times \widetilde{\mathcal{A}}$ be of the form

$$
\begin{array}{ll}
\varphi(z)=z+\sum_{n=2}^{\infty} \alpha_{n} z^{n}, \quad \phi(z)=z+\sum_{n=2}^{\infty} \beta_{n} z^{n} \quad(z \in \mathcal{U}), \\
\chi(z)=z+\sum_{n=2}^{\infty} \gamma_{n} z^{n}, \quad \psi(z)=z+\sum_{n=2}^{\infty} \delta_{n} z^{n} \quad(z \in \mathcal{U}), \\
p(z)=1+\sum_{n=1}^{\infty} p_{n} z^{n}, \quad q(z)=1+\sum_{n=1}^{\infty} q_{n} z^{n} \quad\left(z \in \mathcal{U}, p_{1}, q_{1} \neq 0\right) .
\end{array}
$$

By $\mathcal{W}_{\alpha}(\Phi, \Psi ; p)$ we denote the class of functions $f \in \mathcal{A}$ such that

$$
(\varphi * f)(z)(\chi * f)(z) \neq 0 \quad(z \in \mathcal{U} \backslash\{0\})
$$

and

$$
(1-\alpha) \frac{\phi * f}{\varphi * f}+\alpha \frac{\psi * f}{\chi * f} \prec p .
$$

Moreover, let us put

$$
\begin{aligned}
& \mathcal{W}(\Phi ; p):=\mathcal{W}_{0}(\Phi, \Phi ; p), \quad \mathcal{M}_{\alpha}(\varphi ; p):=\mathcal{W}_{\alpha}\left(\left(z \varphi^{\prime}(z), \varphi\right),\left(z\left(z \varphi^{\prime}(z)\right)^{\prime}, z \varphi^{\prime}(z)\right) ; p\right), \\
& \mathcal{S}^{c}(\varphi ; p):=\mathcal{M}_{1}(\varphi ; p), \quad \mathcal{S}^{*}(\varphi ; p):=\mathcal{M}_{0}(\varphi ; p), \quad \mathcal{S}^{*}(p):=\mathcal{S}^{*}\left(\frac{z}{1-z} ; p\right) .
\end{aligned}
$$


It is clear that the class $\mathcal{M}_{\alpha}(\varphi ; p)$ contains functions $f \in \mathcal{A}$ such that

$$
(1-\alpha) \frac{z(\varphi * f)^{\prime}(z)}{(\varphi * f)(z)}+\alpha\left(1+\frac{z(\varphi * f)^{\prime \prime}(z)}{(\varphi * f)^{\prime}(z)}\right) \prec p(z) .
$$

We denote by $\mathcal{C W}(\Phi ; \mathrm{P})$ the class of functions $f \in \mathcal{A}$ for which there exist a function $g \in$ $\mathcal{S}^{*}(q)$ such that $(\varphi * f)(z) \neq 0(z \in \mathcal{U} \backslash\{0\})$ and

$$
\frac{\phi * f}{\varphi * g} \prec p .
$$

Moreover, let us denote $\mathcal{C W}(\Phi ; p):=\mathcal{C} \mathcal{W}(\Phi ; p, p)$.

In particular, the classes

$$
\mathcal{M}_{\alpha}:=\mathcal{M}_{\alpha}\left(\frac{z}{1-z} ; \frac{1+z}{1-z}\right), \quad \mathcal{S}^{*}:=\mathcal{M}_{0}, \quad \mathcal{S}^{c}:=\mathcal{M}_{1}
$$

are the well-known classes of $\alpha$-convex Mocanu functions [24], starlike functions and convex functions, respectively. The class $\mathcal{C}:=\mathcal{W}\left(\left(\frac{z}{(1-z)^{2}}, \frac{z}{1-z}\right) ; \frac{1+z}{1-z}\right)$ is the well-known class of close-to convex functions with argument $\beta=0$.

The object of the paper is to solve the Fekete-Szegö problem in the defined classes of functions. Moreover, we find sharp bounds for the second and third coefficient in these classes. Some remarks depicting consequences of the main results are also mentioned.

\section{The main results}

The following lemmas will be required in our present investigation.

Lemma 1 [3] If $\omega \in \Omega, \omega(z)=\sum_{n=1}^{\infty} c_{n} z^{n}(z \in \mathcal{U})$, then

$$
\begin{aligned}
& \left|c_{n}\right| \leq 1 \quad(n=1,2), \quad\left|c_{2}\right| \leq 1-\left|c_{1}\right|^{2}, \\
& \left|c_{2}-\mu c_{1}^{2}\right| \leq \max \{1,|\mu|\} \quad(\mu \in \mathbb{C}) .
\end{aligned}
$$

The result is sharp. The functions

$$
\omega(z)=z, \quad \omega_{a}(z)=z \frac{z+a}{1+\bar{a} z} \quad(z \in \mathcal{U},|a|<1)
$$

are the extremal functions.

\section{Theorem 1 Let}

$$
(1-\alpha)\left(\beta_{k}-\alpha_{k}\right)+\alpha\left(\delta_{k}-\gamma_{k}\right) \neq 0 \quad(k=2,3) .
$$

Iff $\in \mathcal{W}_{\alpha}(\Phi, \Psi ; p)$, then

$$
\begin{aligned}
& \left|a_{2}\right| \leq \frac{\left|p_{1}\right|}{\left|(1-\alpha)\left(\beta_{2}-\alpha_{2}\right)+\alpha\left(\delta_{2}-\gamma_{2}\right)\right|}, \\
& \left|a_{3}\right| \leq \frac{\left|p_{1}\right|}{\left|(1-\alpha)\left(\beta_{3}-\alpha_{3}\right)+\alpha\left(\delta_{3}-\gamma_{3}\right)\right|} \max \{1,|\beta|\}, \\
& \left|a_{3}-\mu a_{2}^{2}\right| \leq \frac{\left|p_{1}\right|}{\left|(1-\alpha)\left(\beta_{3}-\alpha_{3}\right)+\alpha\left(\delta_{3}-\gamma_{3}\right)\right|} \max \{1,|\gamma|\} \quad(\mu \in \mathbb{C}),
\end{aligned}
$$


where

$$
\begin{aligned}
& \beta=\frac{p_{2}}{p_{1}}+\frac{(1-\alpha) \alpha_{2}\left(\beta_{2}-\alpha_{2}\right)+\alpha \gamma_{2}\left(\delta_{2}-\gamma_{2}\right)}{\left[(1-\alpha)\left(\beta_{2}-\alpha_{2}\right)+\alpha\left(\delta_{2}-\gamma_{2}\right)\right]^{2}} p_{1}, \\
& \gamma=\frac{(1-\alpha)\left(\beta_{3}-\alpha_{3}\right)+\alpha\left(\delta_{3}-\gamma_{3}\right)}{\left[(1-\alpha)\left(\beta_{2}-\alpha_{2}\right)+\alpha\left(\delta_{2}-\gamma_{2}\right)\right]^{2}} p_{1} \mu-\beta .
\end{aligned}
$$

The results are sharp.

Proof Let $f \in \mathcal{W}_{\alpha}(\Phi, \Psi ; p)$. Then there exists a function $\omega \in \Omega, \omega(z)=\sum_{n=1}^{\infty} c_{n} z^{n}(z \in \mathcal{U})$, such that

$$
(1-\alpha) \frac{\phi * f}{\varphi * f}+\alpha \frac{\psi * f}{\chi * f}=p \circ \omega
$$

It is easy to verify that

$$
\begin{aligned}
& (p \circ \omega)(z)=1+\left(p_{1} c_{1}\right) z+\left(p_{1} c_{2}+p_{2} c_{1}^{2}\right) z^{2}+\cdots \quad(z \in \mathcal{U}) \\
& (1-\alpha) \frac{(\phi * f)(z)}{(\varphi * f)(z)}+\alpha \frac{(\psi * f)(z)}{(\chi * f)(z)}=1+A_{2} z+A_{3} z^{2}+\cdots \quad(z \in \mathcal{U})
\end{aligned}
$$

where

$$
\begin{aligned}
& A_{2}=\left[(1-\alpha)\left(\beta_{2}-\alpha_{2}\right)+\alpha\left(\delta_{2}-\gamma_{2}\right)\right] a_{2}, \\
& A_{3}=\left[(1-\alpha)\left(\beta_{3}-\alpha_{3}\right)+\alpha\left(\delta_{3}-\gamma_{3}\right)\right] a_{3}-\left[(1-\alpha) \alpha_{2}\left(\beta_{2}-\alpha_{2}\right)+\alpha \gamma_{2}\left(\delta_{2}-\gamma_{2}\right)\right] a_{2}^{2} .
\end{aligned}
$$

Thus, by (7), we have

$$
\begin{aligned}
& a_{2}=\frac{p_{1} c_{1}}{(1-\alpha)\left(\beta_{2}-\alpha_{2}\right)+\alpha\left(\delta_{2}-\gamma_{2}\right)}, \\
& a_{3}=\frac{p_{1} c_{2}+p_{2} c_{1}^{2}+\left[(1-\alpha) \alpha_{2}\left(\beta_{2}-\alpha_{2}\right)+\alpha \gamma_{2}\left(\delta_{2}-\gamma_{2}\right)\right] a_{2}^{2}}{(1-\alpha)\left(\beta_{3}-\alpha_{3}\right)+\alpha\left(\delta_{3}-\gamma_{3}\right)},
\end{aligned}
$$

which by Lemma 1 gives sharp estimation (2). Let $\mu$ be a complex number. Then, by (10) and (11) we obtain

$$
a_{3}-\mu a_{2}^{2}=\frac{p_{1}}{(1-\alpha)\left(\beta_{3}-\alpha_{3}\right)+\alpha\left(\delta_{3}-\gamma_{3}\right)}\left\{c_{2}-\gamma c_{1}^{2}\right\},
$$

where $\gamma$ is defined by (6). Thus, by Lemma 1 , we have (4). Let functions $f_{1}, f_{2} \in \mathcal{A}$ satisfy the conditions

$$
\begin{aligned}
& (1-\alpha) \frac{\left(\phi * f_{1}\right)(z)}{\left(\varphi * f_{1}\right)(z)}+\alpha \frac{\left(\psi * f_{1}\right)(z)}{\left(\chi * f_{1}\right)(z)}=p(z) \quad(z \in \mathcal{U}), \\
& (1-\alpha) \frac{\left(\phi * f_{2}\right)(z)}{\left(\varphi * f_{2}\right)(z)}+\alpha \frac{\left(\psi * f_{2}\right)(z)}{\left(\chi * f_{2}\right)(z)}=p\left(z^{2}\right) \quad(z \in \mathcal{U}) .
\end{aligned}
$$

Then the functions belong to the class $\mathcal{W}_{\alpha}(\Phi, \Psi ; p)$ and they realize the equality in the estimation (4). Thus, the results are sharp. Putting $\mu=0$ in (4) we get the sharp estimation (3). 
Theorem 2 Let $\alpha \neq-\frac{1}{2},-1$. Iff $\in \mathcal{M}_{\alpha}(\varphi, p)$, then

$$
\begin{aligned}
& \left|a_{2}\right| \leq \frac{\left|p_{1}\right|}{(1+\alpha)\left|\alpha_{2}\right|}, \quad\left|a_{3}\right| \leq \frac{\left|p_{1}\right|}{2(1+2 \alpha)\left|\alpha_{3}\right|} \max \{1,|\beta|\} \\
& \left|a_{3}-\mu a_{2}^{2}\right| \leq \frac{\left|p_{1}\right|}{2(1+2 \alpha)\left|\alpha_{3}\right|} \max \{1,|\gamma|\} \quad(\mu \in \mathbb{C}),
\end{aligned}
$$

where

$$
\beta=\frac{p_{2}}{p_{1}}+\frac{(1+3 \alpha)}{(1+\alpha)^{2}} p_{1}, \quad \gamma=\frac{2(1+2 \alpha) \alpha_{3} p_{1}}{(1+\alpha)^{2} \alpha_{2}^{2}} \mu-\beta
$$

The results are sharp.

Proof Let $f \in \mathcal{M}_{\alpha}(\varphi, p)=\mathcal{W}_{\alpha}(\Phi, \Psi ; p)$, where

$$
\chi(z)=\phi(z)=z \varphi^{\prime}(z), \quad \psi(z)=z\left(z \varphi^{\prime}(z)\right)^{\prime} \quad(z \in \mathcal{U}) .
$$

Since

$$
\beta_{n}=\gamma_{n}=n \alpha_{n}, \quad \delta_{n}=n^{2} \alpha_{n} \quad(n=2,3),
$$

the results follow from Theorem 1 .

If we put $\alpha=0$ in Theorem 1 , then we obtain the following theorem.

Theorem 3 Let $\beta_{k} \neq \alpha_{k}(k=2,3)$. If $\in \mathcal{W}(\Phi ; p)$, then

$$
\begin{aligned}
& \left|a_{2}\right| \leq \frac{\left|p_{1}\right|}{\left|\beta_{2}-\alpha_{2}\right|}, \quad\left|a_{3}\right| \leq \frac{\left|p_{1}\right|}{\left|\beta_{3}-\alpha_{3}\right|} \max \{1,|\beta|\}, \\
& \left|a_{3}-\mu a_{2}^{2}\right| \leq \frac{\left|p_{1}\right|}{\left|\beta_{3}-\alpha_{3}\right|} \max \{1,|\gamma|\} \quad(\mu \in \mathbb{C}),
\end{aligned}
$$

where

$$
\beta=\frac{p_{2}}{p_{1}}+\frac{\alpha_{2} p_{1}}{\beta_{2}-\alpha_{2}}, \quad \gamma=\frac{\beta_{3}-\alpha_{3}}{\left(\beta_{2}-\alpha_{2}\right)^{2}} p_{1} \mu-\beta .
$$

The results are sharp.

Theorem 4 Let $\beta_{2} \beta_{3} \neq 0$. If $f \in \mathcal{C W}(\Phi ; \mathrm{P})$, then

$$
\begin{aligned}
& \left|a_{3}-\mu a_{2}^{2}\right| \leq \frac{1}{2\left|\beta_{3}\right|}\left(D+\max \left\{0, A_{\mu}\right\}+\max \left\{0, B_{\mu}\right\}\right), \\
& \left|a_{3}\right| \leq \frac{1}{2\left|\beta_{3}\right|}\left(D+\max \left\{0, A_{0}\right\}+\max \left\{0, B_{0}\right\}\right) \\
& \left|a_{2}\right|=2 \frac{\left|p_{1}\right|+\left|\alpha_{2}\right|\left|q_{1}\right|}{\left|\beta_{2}\right|}
\end{aligned}
$$


where

$$
\begin{aligned}
& A_{\mu}=\left|\alpha_{3}\left(q_{2}+q_{1}^{2}\right)-2 \mu \frac{\alpha_{2}^{2} \beta_{3} q_{1}^{2}}{\beta_{2}^{2}}\right|+C_{\mu}-\left|\alpha_{3}\right|\left|q_{1}\right|, \quad D=2\left|p_{1}\right|+\left|\alpha_{3}\right|\left|q_{1}\right|, \\
& B_{\mu}=2\left|p_{2}-\mu \frac{\beta_{3} p_{1}^{2}}{\beta_{2}^{2}}\right|+C_{\mu}-2\left|p_{1}\right|, \quad C_{\mu}=\left|\alpha_{2}\right|\left|p_{1}\right|\left|q_{1}\right|\left|1-2 \mu \frac{\beta_{3}}{\beta_{2}^{2}}\right| .
\end{aligned}
$$

The results (12) and (13) are sharp for $A_{\mu} B_{\mu} \geq 0$ and $A_{0} B_{0} \geq 0$, respectively.

Proof Let $f \in \mathcal{C} \mathcal{W}(\Phi ; \mathrm{P})$. Then there exists a function $g \in S^{*}(q)$ and functions $\omega, \eta \in \Omega$,

$$
\omega(z)=\sum_{n=1}^{\infty} c_{n} z^{n}, \quad \eta(z)=\sum_{n=1}^{\infty} d_{n} z^{n} \quad(z \in \mathcal{U}),
$$

such that

$$
\frac{\phi * f}{\varphi * g}=p \circ \omega, \quad \frac{z g^{\prime}(z)}{g(z)}=(q \circ \eta)(z) \quad(z \in \mathcal{U}) .
$$

Thus, by (8), we have

$$
\begin{aligned}
& b_{2}=q_{1} d_{1}, \quad 2 b_{3}=q_{1} d_{2}+\left(q_{2}+q_{1}^{2}\right) d_{1}^{2}, \quad a_{2}=\frac{c_{1} p_{1}+\alpha_{2} d_{1} q_{1}}{\beta_{2}}, \\
& \beta_{3} a_{3}=\frac{\alpha_{3}}{2}\left\{q_{1} d_{2}+\left(q_{2}+q_{1}^{2}\right) d_{1}^{2}\right\}+\alpha_{2} p_{1} q_{1} c_{1} d_{1}+p_{1} c_{2}+p_{2} c_{1}^{2},
\end{aligned}
$$

and by Lemma 1, we obtain the sharp estimation (14). Let $\mu$ be a complex number. Then, by (18), (19) and Lemma 1 we have

$$
2\left|\beta_{3}\right|\left|a_{3}-\mu a_{2}^{2}\right| \leq(A-C)\left|d_{1}\right|^{2}+(B-C)\left|c_{1}\right|^{2}+2 C\left|d_{1}\right|\left|c_{1}\right|+D,
$$

or equivalently

$$
2\left|\beta_{3}\right|\left|a_{3}-\mu a_{2}^{2}\right| \leq A\left|d_{1}\right|^{2}+B\left|c_{1}\right|^{2}-C\left(\left|d_{1}\right|-\left|c_{1}\right|\right)^{2}+D,
$$

where $A=A_{\mu}, B=B_{\mu}, C=C_{\mu}, D$ are defined by (15) and (16). Thus, we obtain

$$
2\left|\beta_{3}\right|\left|a_{3}-\mu a_{2}^{2}\right| \leq A\left|d_{1}\right|^{2}+B\left|c_{1}\right|^{2}+D,
$$

and, in consequence, by Lemma 1 we have (12). It is easy to verify that the equality in (22) is attained by choosing $c_{1}=d_{1}=1, c_{2}=d_{2}=0$ if $A \geq 0, B \geq 0$ or $c_{1}=d_{1}=0, c_{2}=d_{2}=1$ if $A \leq 0, B \leq 0$. Therefore, we consider functions $f_{1}, f_{2} \in \mathcal{A}$ such that

$$
\frac{\left(\phi * f_{1}\right)(z)}{(\varphi * g)(z)}=p(z), \quad \frac{z g^{\prime}(z)}{g(z)}=q(z) \quad(z \in \mathcal{U})
$$

and

$$
\frac{\left(\phi * f_{2}\right)(z)}{(\varphi * g)(z)}=p\left(z^{2}\right), \quad \frac{z g^{\prime}(z)}{g(z)}=p\left(z^{2}\right) \quad(z \in \mathcal{U}),
$$


respectively. Then the functions belong to the class $\mathcal{C W}(\Phi ; p)$ and they realize the equality in the estimation (12) for $A B \geq 0$. Putting $\mu=0$ in (12), we get the sharp estimation (13).

The following theorem gives the complete sharp estimation of the Fekete-Szegö functional in the class $\mathcal{C W}(\Phi ; \mathrm{P})$.

Theorem 5 Let $\beta_{2} \beta_{3} \neq 0$. If $\in \mathcal{C} \mathcal{W}(\Phi ; \mathrm{P})$, then

$$
\left|a_{3}-\mu a_{2}^{2}\right| \leq\left\{\begin{array}{lc}
\frac{1}{2\left|\beta_{3}\right|}(A+B+D) & \text { if } 0 \leq A \leq C \vee 0 \leq B \leq C \\
& \vee(A \geq C \wedge B \geq C), \\
\frac{D}{2\left|\beta_{3}\right|} & \text { if } A \leq 0 \wedge B \leq 0, \\
\frac{1}{2\left|\beta_{3}\right|}\left(D+B-C+\frac{C^{2}}{C-A}\right) & \text { if } A<0 \wedge B \geq C, \\
\frac{1}{2\left|\beta_{3}\right|}\left(D+A-C+\frac{C^{2}}{C-B}\right) & \text { if } B<0 \wedge A \geq C,
\end{array}\right.
$$

where $A=A_{\mu}, B=B_{\mu}, C=C_{\mu}, D$ are defined by (15) and (16). The result is sharp.

Proof From Theorem 4, we have sharp estimation (23) for $A B \geq 0$. Let now $A \geq C$ and $B<0$. Then, by (20) and Lemma 1 we have

$$
2\left|\beta_{3}\right|\left|a_{3}-\mu a_{2}^{2}\right| \leq v\left(\left|c_{1}\right|\right)
$$

where

$$
v(x):=-(C-B) x^{2}+2 C x+(A-C)+D .
$$

Simply calculations give that the function $v$ attains a maximum in the interval $[0,1]$ at the point $x=\frac{C}{C-B} \leq 1$. Thus, we have (23) for $A \geq C$ and $B<0$. Moreover, the equality in (20) is attained by choosing the functions $\eta(z)=z, \omega(z)=z \frac{z+a}{1+\bar{a} z}$, for $a=\frac{C}{C-B}$, i.e. $c_{1}=\frac{C}{C-B}, d_{1}=1$ and $c_{2}=1-|a|^{2}, d_{2}=0$. Therefore, the result is sharp for $A \geq C$ and $B<0$.

Next, let $A<0$ and $C \leq B$. Then, by (20) and Lemma 1 we have

$$
2\left|\beta_{3}\right|\left|a_{3}-\mu a_{2}^{2}\right| \leq \widetilde{v}\left(\left|d_{1}\right|\right)
$$

where

$$
\widetilde{v}(x):=-(C-A) x^{2}+2 C x+(B-C)+D .
$$

Since the function $\widetilde{v}$ attains a maximum in the interval $[0,1]$ at the point $x=\frac{C}{C-A} \leq 1$, we have the estimation (23) for $A<0$ and $C \leq B$. The equality in (20) is attained by choosing the functions $\omega(z)=z, \eta(z)=z \frac{z+a}{1+\bar{a} z}$, for $a=\frac{C}{C-A}$, i.e. $c_{1}=1, d_{1}=\frac{C}{C-A}$ and $c_{2}=0, d_{2}=1-|a|^{2}$. Finally, let us assume $(0 \leq A \leq C \wedge B \leq 0) \vee(0 \leq B \leq C \wedge A \leq 0)$. Then, by (20) we have

$$
2\left|\beta_{3}\right|\left|a_{3}-\mu a_{2}^{2}\right| \leq F\left(\left|c_{1}\right|,\left|d_{1}\right|\right)
$$

where

$$
F(x, y)=-(C-A) x^{2}-(C-B) y^{2}+2 C x y+D .
$$


Since $F$ is the continuous function on $T:=[0,1] \times[0,1]$, by $(24)$ we have

$$
2\left|\beta_{3}\right|\left|a_{3}-\mu a_{2}^{2}\right| \leq \max F(T)=\max F(\partial T \cup K)
$$

where $K$ is the set of critical points of the function $F$ in $T$. It is easy to verify that

$$
K \backslash \partial T= \begin{cases}\varnothing & \text { if } C^{2} \neq(C-A)(C-B) \vee A=C, \\ \left\{(x, y) \in \operatorname{int} T: x=\frac{C}{C-A} y\right\} & \text { if } C^{2}=(C-A)(C-B) \wedge A \neq C .\end{cases}
$$

If $C^{2}=(A-C)(B-C) \neq 0$, then

$$
F\left(\frac{C}{C-A} y, y\right)=\frac{C^{2}-(C-A)(C-B)}{A-C} y^{2}+D=D \quad(y \in[0,1]) .
$$

Moreover, we have

$$
\begin{aligned}
& F(x, 0)=-(C-A) x^{2}+D \leq D, \quad F(0, y)=-(C-B) y^{2}+D \leq D \quad(x, y \in[0,1]), \\
& F(x, 1)=-(C-A) x^{2}+2 C x+B-C+D \leq F(1,1)=A+B+D \quad(x \in[0,1]), \\
& F(1, y)=-(C-B) y^{2}+2 C y+A-C+D \leq F(1,1)=A+B+D \quad(y \in[0,1]) .
\end{aligned}
$$

Thus, we obtain

$$
\max F(\partial T \cup K)=A+B+D
$$

which by (25) gives (23) for $(0 \leq A \leq C \wedge B \leq 0) \vee(0 \leq B \leq C \wedge A \leq 0)$. The equality in (24) is attained by choosing $c_{1}=d_{1}=1$ and $c_{2}=d_{2}=0$. Therefore, the result is sharp and the proof is completed.

Putting $\mu=0$ in Theorem 5 we obtain the following theorem.

Theorem 6 Let $\beta_{2} \beta_{3} \neq 0$. Iff $\in \mathcal{C W}(\Phi ; \mathrm{P})$, then

$$
\left|a_{3}\right| \leq\left\{\begin{array}{lrl}
\frac{1}{2\left|\beta_{3}\right|}(A+B+D) & \text { if } 0 \leq A \leq C \vee 0 \leq B \leq C \\
& \vee(A \geq C \wedge B \geq C), \\
\frac{D}{2\left|\beta_{3}\right|} & \text { if } A \leq 0 \wedge B \leq 0, \\
\frac{1}{2\left|\beta_{3}\right|}\left(D+B-C+\frac{C^{2}}{C-A}\right) & \text { if } A<0 \wedge B \geq C, \\
\frac{1}{2\left|\beta_{3}\right|}\left(D+A-C+\frac{C^{2}}{C-B}\right) & \text { if } B<0 \wedge A \geq C,
\end{array}\right.
$$

where $A=A_{0}, B=B_{0}, C=C_{0}, D$ are defined by (15) and (16). The result is sharp.

\section{Applications}

If we put $\alpha=1$ and $\alpha=0$ in Theorem 2 , then we obtain the following two corollaries.

Corollary 1 Let $\alpha_{2} \alpha_{3} \neq 0$. If $\in \in \mathcal{S}^{c}(\varphi, p)$, then

$$
\left|a_{2}\right| \leq \frac{1}{2}\left|\frac{p_{1}}{\alpha_{2}}\right|, \quad\left|a_{3}\right| \leq \frac{1}{6}\left|\frac{p_{1}}{\alpha_{3}}\right| \max \left\{1,\left|\frac{p_{2}}{p_{1}}+p_{1}\right|\right\},
$$




$$
\left|a_{3}-\mu a_{2}^{2}\right| \leq \frac{1}{6}\left|\frac{p_{1}}{\alpha_{3}}\right| \max \{1,|\gamma|\} \quad(\mu \in \mathbb{C})
$$

where

$$
\gamma=\frac{3 \alpha_{3} p_{1}}{2 \alpha_{2}^{2}} \mu-p_{1}-\frac{p_{2}}{p_{1}} .
$$

The results are sharp.

Corollary 2 Let $\alpha_{2} \alpha_{3} \neq 0$. If $f \in \mathcal{S}^{*}(\varphi, p)$, then

$$
\begin{aligned}
& \left|a_{2}\right| \leq\left|\frac{p_{1}}{\alpha_{2}}\right|, \quad\left|a_{3}\right| \leq \frac{1}{2}\left|\frac{p_{1}}{\alpha_{3}}\right| \max \left\{1,\left|\frac{p_{2}}{p_{1}}+p_{1}\right|\right\}, \\
& \left|a_{3}-\mu a_{2}^{2}\right| \leq \frac{1}{2}\left|\frac{p_{1}}{\alpha_{3}}\right| \max \{1,|\gamma|\} \quad(\mu \in \mathbb{C}),
\end{aligned}
$$

where

$$
\gamma=2 \frac{\alpha_{3} p_{1}}{\alpha_{2}^{2}} \mu-p_{1}-\frac{p_{2}}{p_{1}} .
$$

The results are sharp.

Choosing the function $p$ in Theorems 1-6, we can obtain several new results.

Let $a, b$ be complex number, $|b|<1, a \neq b$, and let

$$
p(z)=\frac{1+a z}{1+b z} \quad(z \in \mathcal{U}) .
$$

It is clear, that

$$
p(z)=1+(a-b) z-b(a-b) z^{2}+\cdots \quad(z \in \mathcal{U}) .
$$

Thus, by Theorems 1-3 and 5, we obtain the following four corollaries.

Corollary 3 Let $(1-\alpha)\left(\beta_{k}-\alpha_{k}\right)+\alpha\left(\delta_{k}-\gamma_{k}\right) \neq 0\left(k=2\right.$, 3). Iff $\in \mathcal{W}_{\alpha}\left(\Phi, \Psi ; \frac{1+a z}{1+b z}\right)$, then

$$
\begin{aligned}
& \left|a_{2}\right| \leq \frac{|a-b|}{\left|(1-\alpha)\left(\beta_{2}-\alpha_{2}\right)+\alpha\left(\delta_{2}-\gamma_{2}\right)\right|}, \\
& \left|a_{3}\right| \leq \frac{|a-b|}{\left|(1-\alpha)\left(\beta_{3}-\alpha_{3}\right)+\alpha\left(\delta_{3}-\gamma_{3}\right)\right|} \max \{1,|\beta|\}, \\
& \left|a_{3}-\mu a_{2}^{2}\right| \leq \frac{|a-b|}{\left|(1-\alpha)\left(\beta_{3}-\alpha_{3}\right)+\alpha\left(\delta_{3}-\gamma_{3}\right)\right|} \max \{1,|\gamma|\} \quad(\mu \in \mathbb{C}),
\end{aligned}
$$

where

$$
\begin{aligned}
& \beta=-b+\frac{(1-\alpha) \alpha_{2}\left(\beta_{2}-\alpha_{2}\right)+\alpha \gamma_{2}\left(\delta_{2}-\gamma_{2}\right)}{\left[(1-\alpha)\left(\beta_{2}-\alpha_{2}\right)+\alpha\left(\delta_{2}-\gamma_{2}\right)\right]^{2}}(a-b), \\
& \gamma=\frac{(1-\alpha)\left(\beta_{3}-\alpha_{3}\right)+\alpha\left(\delta_{3}-\gamma_{3}\right)}{\left[(1-\alpha)\left(\beta_{2}-\alpha_{2}\right)+\alpha\left(\delta_{2}-\gamma_{2}\right)\right]^{2}}(a-b) \mu-\beta .
\end{aligned}
$$

The results are sharp. 
Corollary 4 Let $\alpha \neq-\frac{1}{2},-1$. If $\in \mathcal{M}_{\alpha}\left(\varphi, \frac{1+a z}{1+b z}\right)$, then

$$
\begin{aligned}
& \left|a_{2}\right| \leq \frac{|a-b|}{|1+\alpha|\left|\alpha_{2}\right|}, \quad\left|a_{3}\right| \leq \frac{|a-b|}{2|1+2 \alpha|\left|\alpha_{3}\right|} \max \{1,|\beta|\}, \\
& \left|a_{3}-\mu a_{2}^{2}\right| \leq \frac{|a-b|}{2|1+2 \alpha|\left|\alpha_{3}\right|} \max \{1,|\gamma|\} \quad(\mu \in \mathbb{C}),
\end{aligned}
$$

where

$$
\beta=-b+\frac{(1+3 \alpha)}{(1+\alpha)^{2}}(a-b), \quad \gamma=\frac{2(1+2 \alpha)(a-b) \alpha_{3}}{(1+\alpha)^{2} \alpha_{2}^{2}} \mu-\beta
$$

The results are sharp.

Corollary 5 Let $\beta_{k} \neq \alpha_{k}(k=2,3)$. Iff $\in \mathcal{W}\left(\Phi ; \frac{1+a z}{1+b z}\right)$, then

$$
\begin{aligned}
& \left|a_{2}\right| \leq \frac{|a-b|}{\left|\beta_{2}-\alpha_{2}\right|}, \quad\left|a_{3}\right| \leq \frac{|a-b|}{\left|\beta_{3}-\alpha_{3}\right|} \max \{1,|\beta|\}, \\
& \left|a_{3}-\mu a_{2}^{2}\right| \leq \frac{|a-b|}{\left|\beta_{3}-\alpha_{3}\right|} \max \{1,|\gamma|\} \quad(\mu \in \mathbb{C}),
\end{aligned}
$$

where

$$
\beta=-b+\frac{(a-b) \alpha_{2}}{\beta_{2}-\alpha_{2}}, \quad \gamma=\frac{\left(\beta_{3}-\alpha_{3}\right)(a-b)}{\left(\beta_{2}-\alpha_{2}\right)^{2}} \mu-\beta .
$$

The results are sharp.

Corollary 6 Let $\beta_{2} \beta_{3} \neq 0$. Iff $\in \mathcal{C} \mathcal{W}\left(\Phi ; \frac{1+a z}{1+b z}\right)$, then

$$
\left|a_{3}-\mu a_{2}^{2}\right| \leq \begin{cases}\frac{|b-a|}{2\left|\beta_{3}\right|}(D+A+B) & \text { if } 0 \leq A \leq C \vee 0 \leq B \leq C \\ & \vee(A \geq C \wedge B \geq C), \\ \frac{|b-a|}{2\left|\beta_{3}\right|} D & \text { if } A \leq 0 \wedge B \leq 0, \\ \frac{|b-a|}{2\left|\beta_{3}\right|}\left(D+B-C+\frac{C^{2}}{C-A}\right) & \text { if } A<0 \wedge B \geq C, \\ \frac{|b-a|}{2\left|\beta_{3}\right|}\left(D+A-C+\frac{C^{2}}{C-B}\right) & \text { if } B<0 \wedge A \geq C,\end{cases}
$$

where

$$
\begin{aligned}
& A=\left|\alpha_{3}(2 b-a)-2 \mu \frac{\alpha_{2}^{2} \beta_{3}(b-a)}{\beta_{2}^{2}}\right|+C-\left|\alpha_{3}\right|, \quad D=2+\left|\alpha_{3}\right|, \\
& B=2\left|b-\mu \frac{\beta_{3}(b-a)}{\beta_{2}^{2}}\right|+C-2, \quad C=\left|\alpha_{2}\right||b-a|\left|1-2 \mu \frac{\beta_{3}}{\beta_{2}^{2}}\right| .
\end{aligned}
$$

The result is sharp.

Let $0<\theta \leq 1$ and let

$$
p(z)=\left(\frac{1+z}{1-z}\right)^{\theta} \quad(z \in \mathcal{U})
$$


It is easy to verify, that

$$
p(z)=1+2 \theta z+\theta(\theta+1) z^{2}+\cdots \quad(z \in \mathcal{U}) .
$$

Thus, by Theorems 1-3 and 5, we obtain the following four corollaries.

Corollary 7 Let $(1-\alpha)\left(\beta_{k}-\alpha_{k}\right)+\alpha\left(\delta_{k}-\gamma_{k}\right) \neq 0(k=2,3)$. Iff $\in \mathcal{W}_{\alpha}\left(\Phi, \Psi ;\left(\frac{1+z}{1-z}\right)^{\theta}\right)$, then

$$
\begin{aligned}
& \left|a_{2}\right| \leq \frac{2 \theta}{\left|(1-\alpha)\left(\beta_{2}-\alpha_{2}\right)+\alpha\left(\delta_{2}-\gamma_{2}\right)\right|}, \\
& \left|a_{3}\right| \leq \frac{2 \theta}{\left|(1-\alpha)\left(\beta_{3}-\alpha_{3}\right)+\alpha\left(\delta_{3}-\gamma_{3}\right)\right|} \max \{1,|\beta|\}, \\
& \left|a_{3}-\mu a_{2}^{2}\right| \leq \frac{2 \theta}{\left|(1-\alpha)\left(\beta_{3}-\alpha_{3}\right)+\alpha\left(\delta_{3}-\gamma_{3}\right)\right|} \max \{1,|\gamma|\} \quad(\mu \in \mathbb{C}),
\end{aligned}
$$

where

$$
\begin{aligned}
& \beta=\frac{1+\theta}{2}+2 \frac{(1-\alpha) \alpha_{2}\left(\beta_{2}-\alpha_{2}\right)+\alpha \gamma_{2}\left(\delta_{2}-\gamma_{2}\right)}{\left[(1-\alpha)\left(\beta_{2}-\alpha_{2}\right)+\alpha\left(\delta_{2}-\gamma_{2}\right)\right]^{2}} \theta, \\
& \gamma=2 \frac{(1-\alpha)\left(\beta_{3}-\alpha_{3}\right)+\alpha\left(\delta_{3}-\gamma_{3}\right)}{\left[(1-\alpha)\left(\beta_{2}-\alpha_{2}\right)+\alpha\left(\delta_{2}-\gamma_{2}\right)\right]^{2}} \theta \mu-\beta .
\end{aligned}
$$

The results are sharp.

Corollary 8 Let $\alpha \neq-\frac{1}{2}$, -1 . Iff $\in \mathcal{M}_{\alpha}\left(\varphi,\left(\frac{1+z}{1-z}\right)^{\theta}\right)$, then

$$
\begin{aligned}
& \left|a_{2}\right| \leq \frac{2 \theta}{\left|(1+\alpha) \alpha_{2}\right|}, \quad a_{3} \leq \frac{\theta}{\left|(1+2 \alpha) \alpha_{3}\right|} \max \{1,|\beta|\} \\
& \left|a_{3}-\mu a_{2}^{2}\right| \leq \frac{\theta}{\left|(1+2 \alpha) \alpha_{3}\right|} \max \{1,|\gamma|\} \quad(\mu \in \mathbb{C}),
\end{aligned}
$$

where

$$
\beta=\frac{1+\theta}{2}+\frac{2(1+3 \alpha)}{(1+\alpha)^{2}} \theta, \quad \gamma=\frac{4(1+2 \alpha) \theta \alpha_{3}}{(1+\alpha)^{2} \alpha_{2}^{2}} \mu-\beta
$$

The results are sharp.

Corollary 9 Let $\beta_{k} \neq \alpha_{k}(k=2,3)$. If $\in \mathcal{W}\left(\Phi ;\left(\frac{1+z}{1-z}\right)^{\theta}\right)$, then

$$
\begin{aligned}
& \left|a_{2}\right| \leq \frac{2 \theta}{\left|\beta_{2}-\alpha_{2}\right|}, \quad\left|a_{3}\right| \leq \frac{2 \theta}{\left|\beta_{3}-\alpha_{3}\right|} \max \{1,|\beta|\}, \\
& \left|a_{3}-\mu a_{2}^{2}\right| \leq \frac{2 \theta}{\left|\beta_{3}-\alpha_{3}\right|} \max \{1,|\gamma|\} \quad(\mu \in \mathbb{C}),
\end{aligned}
$$

where

$$
\beta=\frac{1+\theta}{2}+\frac{2 \alpha_{2} \theta}{\beta_{2}-\alpha_{2}}, \quad \gamma=\frac{2\left(\beta_{3}-\alpha_{3}\right) \theta}{\left(\beta_{2}-\alpha_{2}\right)^{2}} \mu-\beta .
$$

The results are sharp. 
Corollary 10 Let $\beta_{2} \beta_{3} \neq 0$. Iff $\in \mathcal{C W}\left(\Phi ;\left(\frac{1+z}{1-z}\right)^{\theta}\right)$, then

$$
\left|a_{3}-\mu a_{2}^{2}\right| \leq\left\{\begin{array}{lc}
\frac{\theta}{\left|\beta_{3}\right|}(D+A+B) & \text { if } 0 \leq A \leq C \vee 0 \leq B \leq C \\
& \vee(A \geq C \wedge B \geq C), \\
\frac{\theta D}{\left|\beta_{3}\right|} & \text { if } A \leq 0 \wedge B \leq 0, \\
\left.\frac{\theta}{\left|\beta_{3}\right|}\left(D+B-C+\frac{C^{2}}{C-A}\right)\right) & \text { if } A<0 \wedge B \geq C, \\
\frac{\theta}{\left|\beta_{3}\right|}\left(D+A-C+\frac{C^{2}}{C-B}\right) & \text { if } B<0 \wedge A \geq C,
\end{array}\right.
$$

where

$$
\begin{aligned}
& A=\left|\alpha_{3} \frac{1+5 \theta}{2}-4 \mu \frac{\alpha_{2}^{2} \beta_{3} \theta}{\beta_{2}^{2}}\right|+C-\left|\alpha_{3}\right|, \quad D=2+\left|\alpha_{3}\right|, \\
& B=\left|1+\theta-4 \mu \frac{\beta_{3} \theta}{\beta_{2}^{2}}\right|+C-2, \quad C=2 \theta\left|\alpha_{2}\right|\left|1-2 \mu \frac{\beta_{3}}{\beta_{2}^{2}}\right| .
\end{aligned}
$$

The result is sharp.

Let $\Omega_{k}=\left\{u+i v: u>k \sqrt{(u-1)^{2}+v^{2}}\right\}, k>0$. Note that $\Omega_{k}$ is the convex domain contained in the right half plane, with $1 \in \Omega_{k}$. More precisely, it is the elliptic domain for $k>1$, the hyperbolic domain for $0<k<1$ and the parabolic domain for $k=1$.

Let us denote by $h_{k}$ the univalent function, which maps the unit disc $\mathcal{U}$ onto the conic domain $\Omega_{k}$ with $h_{k}(0)=1$. Obviously, the function $h_{k}$ is convex in $\mathcal{U}$. It is easy to check that $f \in \mathcal{W}\left(\Phi ; h_{k}\right)$ if and only if

$$
\operatorname{Re}\left(\frac{(\phi * f)(z)}{(\varphi * g)(z)}\right)>k\left|\frac{(\phi * f)(z)}{(\varphi * g)(z)}-1\right| \quad(z \in \mathcal{U})
$$

The following lemma gives coefficients estimates for the function.

Lemma 2 [13] Let $h_{k}=1+\sum_{n=1}^{\infty} p_{n} z^{n}(z \in \mathcal{U})$. Then

$$
\begin{gathered}
p_{1}= \begin{cases}\frac{2 D^{2}(k)}{1-k^{2}} & \text { for } 0 \leq k<1, \\
\frac{8}{\pi^{2}} & \text { for } k=1, \\
\frac{\pi^{2}}{4 \sqrt{t}(1+t)\left(k^{2}-1\right) \mathcal{K}^{2}(t)} & \text { for } k>1,\end{cases} \\
p_{2}= \begin{cases}\frac{D^{2}(k)+2}{3} p_{1} & \text { for } 0 \leq k<1, \\
\frac{2}{3} p_{1} & \text { for } k=1, \\
\frac{4\left(t^{2}+6 t+1\right) \mathcal{K}^{2}(t)-\pi^{2}}{24 \sqrt{t}(1+t) \mathcal{K}^{2}(t)} p_{1} & \text { for } k>1,\end{cases}
\end{gathered}
$$

where $D(k)=\frac{2}{\pi} \arcsin k$ and $\mathcal{K}^{2}(t)$ is the complete elliptic integral of first kind.

Using Lemma 1 in Theorems 1-5 we obtain the solutions of the Fekete-Szegö problem for the classes $\mathcal{W}_{\alpha}\left(\Phi, \Psi ; h_{k}\right), \mathcal{M}_{\alpha}\left(\varphi, h_{k}\right), \mathcal{W}_{\alpha}\left(\Phi ; h_{k}\right), \mathcal{C W}_{\alpha}\left(\Phi ; h_{k}\right)$.

Remark 1 The classes $\mathcal{W}_{\alpha}(\Phi, \Psi ; h), \mathcal{M}_{\alpha}(\varphi, h), \mathcal{C W}_{\alpha}(\Phi ; \mathrm{P})$ reduced to well-known subclasses by judicious choices of the parameters; see, for example [1-28]. In particular, they 
generalize several well-known classes defined by linear operators, which were investigated in earlier works. Also, the obtained results generalize several results obtained in these classes of functions.

\section{Competing interests}

The author declares that they have no competing interests.

\section{Acknowledgements}

Dedicated to Professor Hari M Srivastava.

Received: 24 January 2013 Accepted: 9 April 2013 Published: 22 April 2013

\section{References}

1. Fekete, M, Szegö, G: Eine Bemerkung über ungerade schlichte Funktionen. J. Lond. Math. Soc. 8, 85-89 (1933)

2. Pfluger, A: The Fekete-Szegö inequality for complex parameters. Complex Var. Theory Appl. 7, 149-160 (1986)

3. Keogh, FR, Merkes, EP: A coefficient inequality for certain classes of analytic functions. Proc. Am. Math. Soc. 20, 8-12 (1969)

4. Ma, W, Minda, D: An internal geometric characterization of strongly starlike functions. Ann. Univ. Mariae Curie-Sklodowska, Sect. A 45, 89-97 (1991)

5. Ma, W, Minda, D: Coefficient inequalities for strongly close-to-convex functions. J. Math. Anal. Appl. 205, 537-553 (1997)

6. Abdel-Gawad, HR, Thomas, DK: The Fekete-Szegö problem for strongly close-to-convex functions. Proc. Am. Math. Soc. 114, 345-349 (1992)

7. Cho, NE, Owa, S: On the Fekete-Szegö and argument inequalities for strongly close-to-star functions. Math. Inequal. Appl. 5, 697-705 (2002)

8. Choi, JH, Kim, YC, Sugawa, T: A general approach to the Fekete-Szegö problem. J. Math. Soc. Jpn. 59(3), 707-727 (2007)

9. Bhowmik, B, Ponnusamy, S, Wirths, K-J: On the Fekete-Szegö problem for concave univalent functions. J. Math. Anal. Appl. 373, 432-438 (2011)

10. Darus, M, Thomas, DK: On the Fekete-Szegö theorem for close-to-convex functions. Math. Jpn. 44, 507-511 (1996)

11. Darus, M, Shanmugam, TN, Sivasubramanian, S: Fekete-Szegö inequality for a certain class of analytic functions. Mathematica 49, 29-34 (2007)

12. Jakubowski, ZJ, Zyskowska, K: On an estimate of a functional in the class of holomorphic univalent functions. Math. Bohem. 118(3), 281-296 (1993)

13. Kanas, S: Coefficient estimates in subclasses of the Carathéodory class related to conic domains. Acta Math. Univ. Comen. 74, 149-161 (2005)

14. Kanas, S, Darwish, HE: Fekete-Szegö problem for starlike and convex functions of complex order. Appl. Math. Lett. 23, 777-782 (2010)

15. Koepf, V: On the Fekete-Szegö problem for close-to-convex functions. Proc. Am. Math. Soc. 101, $89-95$ (1987)

16. Koepf, W: On the Fekete-Szegö problem for close-to-convex functions II. Arch. Math. 49, 420-433 (1987)

17. London, RR: Fekete-Szegö inequalities for close-to-convex functions. Proc. Am. Math. Soc. 117, $947-950$ (1993)

18. Mishra, AK, Gochhayat, P: The Fekete-Szegö problem for $k$-uniformly convex functions and for a class defined by the Owa-Srivastava operator. J. Math. Anal. Appl. 347, 563-572 (2008)

19. Orhan, H, Deniz, E, Răducanu, D: The Fekete-Szegö problem for subclasses of analytic functions defined by a differential operator related to conic domains. Comput. Math. Appl. 59, 283-295 (2010)

20. Orhan, H, Răducanu, D: Fekete-Szegö problem for strongly starlike functions associated with generalized hypergeometric functions. Math. Comput. Model. 50, 430-438 (2009)

21. Sim, YJ, Kwon, OS, Cho, NE, Srivastava, HM: Some classes of analytic functions associated with conic regions. Taiwan. J. Math. 16, 387-408 (2012)

22. Srivastava, HM, Mishra, AK: Applications of fractional calculus to parabolic starlike and uniformly convex functions. Comput. Math. Appl. 39, 57-69 (2000)

23. Srivastava, HM, Mishra, AK, Das, MK: The Fekete-Szegö problem for a subclass of close-to-convex functions. Complex Var. Theory Appl. 44, 145-163 (2001)

24. Mocanu, PT: Une propriété de convexité g énéralisée dans la théorie de la représentation conforme. Mathematica (Cluj) 11(34), 127-133 (1969)

25. Aouf, MK, Dziok, J: Distortion and convolutional theorems for operators of generalized fractional calculus involving Wright function. J. Appl. Anal. 14(2), 183-192 (2008)

26. Dziok, J: Applications of the Jack lemma. Acta Math. Hung. 105, 93-102 (2004)

27. Dziok, J: Classes of functions defined by certain differential-integral operators. J. Comput. Appl. Math. 105, 245-255 (1999)

28. Dziok, J: Inclusion relationships between classes of functions defined by subordination. Ann. Pol. Math. 100, 193-202 (2011)

doi:10.1186/1687-2770-2013-98

Cite this article as: Dziok: A general solution of the Fekete-Szegö problem. Boundary Value Problems 2013 2013:98. 